http://revistas.ucr.ac.cr/index.php/ingenieria www.ucr.ac.cr / ISSN 1409-2441
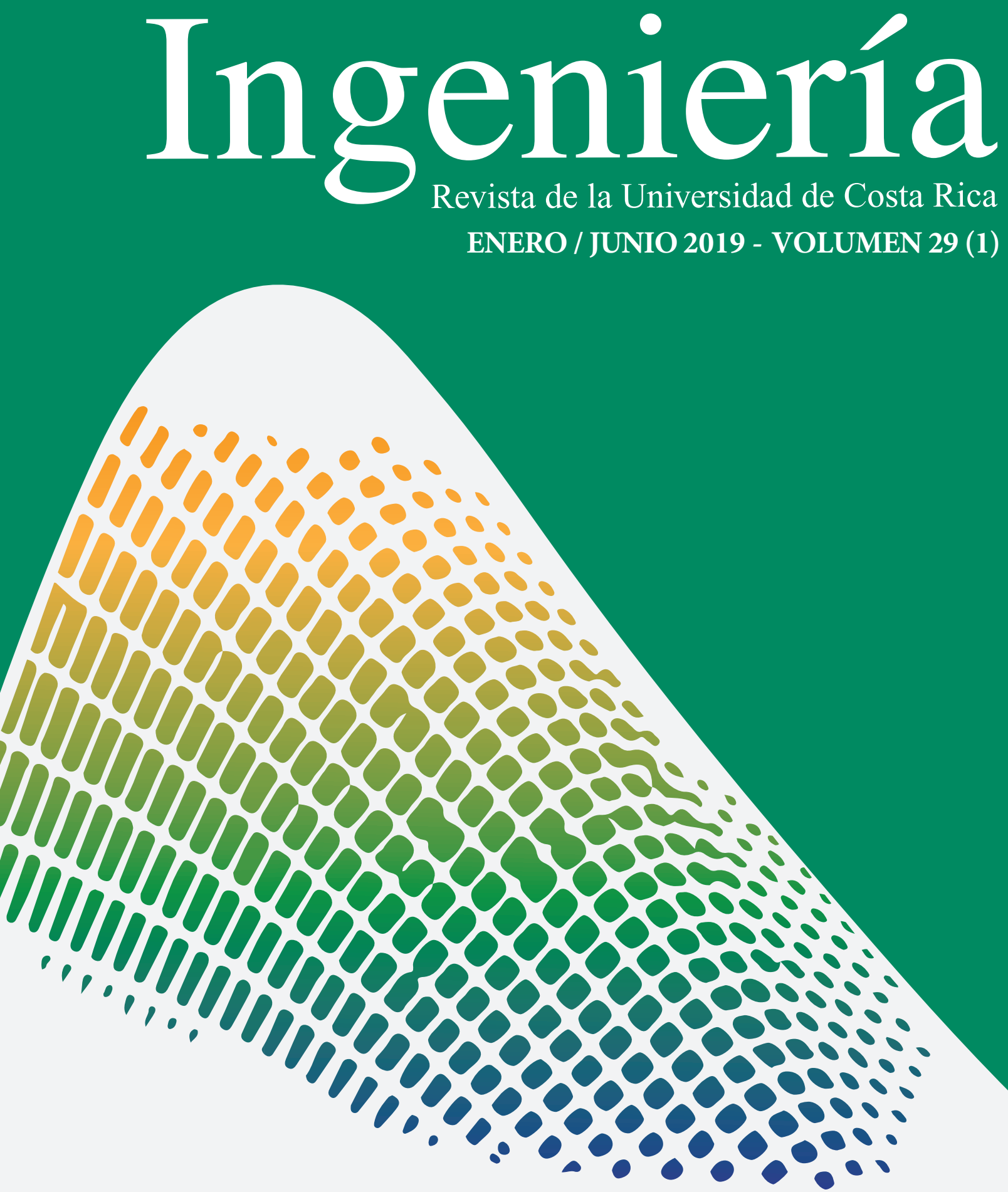


\title{
Estudio de dispersiones de un sistema de parafina-agua-surfactante
}

\author{
A Study of dispersions for a \\ paraffin-water-surfactant system
}

\author{
César Augusto Bernal Samaniego, \\ Universidad de Panamá, Panamá. Nano Dispersions Technology, Inc \\ cesarbernal10@gmail.com \\ Karina María Rodríguez Mora, \\ Universidad de Costa Rica, San José, Costa Rica \\ Instituto de Investigaciones en Ingeniería \\ karina.rodriguezmora@ucr.ac.cr
}

Recibido: 3 de junio 2018

Aceptado: 15 de noviembre 2018

\section{Resumen}

Se estudió el sistema de parafina-aceite-surfactante con la finalidad de obtener emulsiones que tuvieran la capacidad de ser autodispersables en agua y se realizaran con un método de mezclado de baja energía. Se llevó a cabo un primer barrido de formulación con diferentes surfactantes de la línea tween y span, así como las mezclas de los mismos, para la obtención de emulsiones de tipo W/O (agua en aceite, por sus siglas en inglés). Seguidamente, se realizó un segundo barrido de formulación variando el HLB y/o ligeramente el porcentaje de surfactante o el agua empleadas. Posteriormente, se estudió el tamaño de gota y la estabilidad, demostrando que en los sistemas estudiados se obtienen emulsiones más estables con altas cantidades de surfactante y un HLB de 8.

Palabras clave:

Emulsión, estabilidad, HLB, autodispersabilidad.

\begin{abstract}
The propose to study paraffin-water-surfactant was to obtain emulsions that can selfdisperse in water, and which can be made through a low-energy mixing procedure. A first emulsion formulation was carried out with different Tween and Span surfactants, as well as a mixture for obtaining the W/O emulsions (water in oil). Subsequently, a second emulsion formulation was fulfilled, but this time changing the Hydrophilic-Lipophilic Balance (HLB) and/or moderately changing the surfactant or water percentage. Afterwards, the drop size
\end{abstract}


and the stability were studied, and it revealed that more stable emulsions are obtained in the systems aforementioned using larger amounts of surfactants and a HLB 8.

\section{Keywords:}

Emulsion, stability, HLB, self-dispersibility. 


\section{INTRODUCCIÓN}

En primera instancia, es importante tener claro que una emulsión consiste en dos fases inmiscibles, líquido-líquido. Sin embargo, son sistemas termodinámicamente inestables y sus propiedades dependen de la naturaleza de sus componentes y del historial de preparación (histéresis) (Meliky Fogler, 1988; Weiner, 1974). Estas emulsiones se encuentran compuestas por la fase interna, también llamada discontinua, y la externa, o continua. Asimismo, es importante destacar que esos sistemas son termodinámicamente inestables. Por lo tanto, es el surfactante el que asegura determinada estabilidad con respecto a la coalescencia, floculación y flotabilidad (Verbeken, 2005).

Por su parte, las emulsiones se pueden clasificar de acuerdo al tamaño de gota; cuando tienen el tamaño suficiente como para sedimentar, son llamadas macroemulsiones, o simplemente emulsiones. Dicho tamaño es cercano a $1 \mu \mathrm{m}$ de diámetro, valor que corresponde con el límite de observación a través de un microscopio de luz. Por otra parte, se tienen las miniemulsiones o nanoemulsiones, las cuales presentan un diámetro menor de $0,5 \mu \mathrm{m}$. Debido a su tamaño se pueden ver traslúcidas y presentan mayor área superficial; por consiguiente, más cantidad de surfactante para estabilizarla (Piorkowski y McClements, 2013).

Las emulsiones se pueden clasificar de acuerdo a la naturaleza de cada una de sus fases. Pues cuando se tienen gotas de agua dispersas en aceite, o también llamada fase oleosa, se suele expresar como emulsión A/O (fase acuosa dispersa en la oleosa), o también conocida como W/O por sus siglas en inglés Water in Oil. En el caso contrario, se tendría gotas de aceite dispersas en la fase acuosa por tanto sería O/A o O/W, tal y como se puede observar en la Figura 1 (Morrison y Ross, 2002; Salager, 1999).

Por otra parte, se debe destacar que también existe una clasificación de acuerdo con la complejidad de la emulsión, en donde se consideran emulsiones simples a las descritas anteriormente, pero se genera la clasificación de emulsiones múltiple, las cuales presentan varias fases. Lo anterior se ilustra cuando se pueden ver gotas de agua dentro de las gotas de aceite y como fase externa agua, esta sería descrita como W1/O/W2, de igual forma se puede tener el caso contrario $\mathrm{O} 1 / \mathrm{W} / \mathrm{O} 2$, o en dado caso emulsiones aún más complejas, como se muestra en la figura 2. También existe el caso cuando la fase más interna (W1) y la fase más externa (W2) no son iguales, cuando esto sucede, la diferencia de potencial químico entre ambas podría producir una transferencia de masa, fenómeno altamente ventajoso para aplicaciones en dispersiones de difusión controlada (Muguet et al., 2001).

La mezcla apropiada de componentes para producir un sistema, con el fin de llevar a cabo una función determinada, se le llama formulación. Existen una serie de parámetros a tomar en consideración al momento de formular, llamadas variables de formulación, que pueden ser: la naturaleza de los componentes, las proporciones de los componentes y el protocolo de mezclado (Salager, 1999). Un factor determinante en la formulación es el HLB (Hydrophilic-Lipophilic Balance), el cual es una medida del grado de afinidad hidrofílico o lipofílico que posee el surfactante. 
Por su parte, la estabilidad de las emulsiones puede determinarse mediante la medición del tamaño de gota de la misma, la cual se puede ver afectada por fenómenos como índice de flotabilidad, floculación, coalescencia y sedimentación. Asimismo, las emulsiones se pueden utilizar como vehículos de transporte de ingredientes activos, ya sea en el área de la medicina, como lo es en transporte de fármacos, o en la industria agrícola para encapsulación de pesticidas, fertilizantes o biocontroladores. Existen varios estudios en el área médica con aplicaciones terapéuticas, pues de esta forma no solo se tienen sistemas de liberación controlada, sino que se obtienen ventajas, por ejemplo el aumento de biodisponibilidad local al usarlo en sistemas oculares, manteniendo el efecto farmacológico y disminuyendo efectos sistémicos (Tamilvanan, 2004).

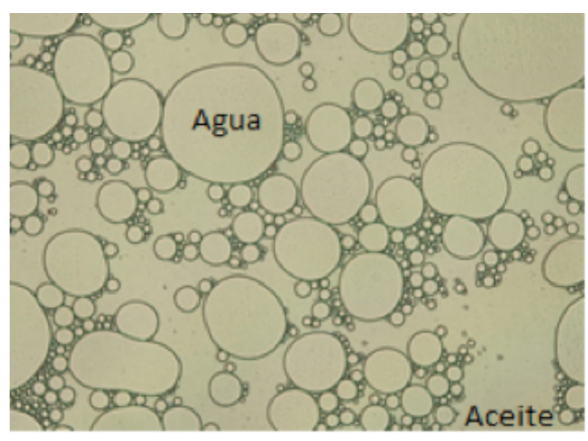

a) Emulsiones agua en aceite $(\mathrm{W} / \mathrm{O})$

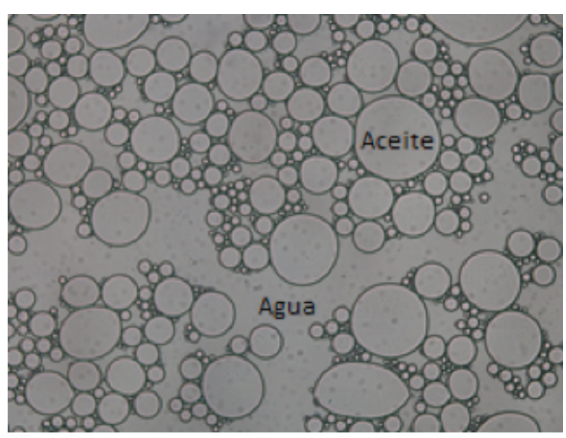

b) Emulsiones aceite en agua $(\mathrm{O} / \mathrm{W})$

Figura 1. Tipos de a emulsión de acuerdo a la composición de las fase continua y discontinua. Nota: Fuente propia del estudio.

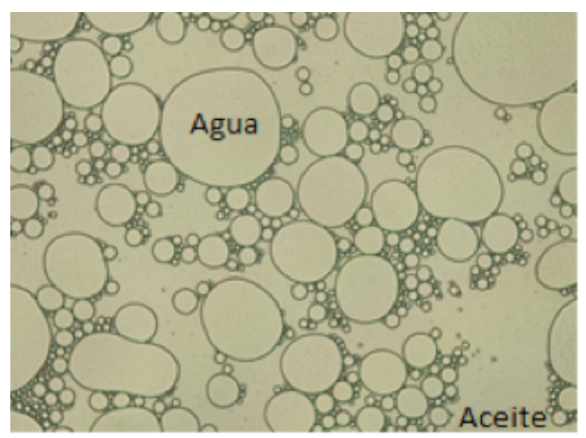

a) Simple W/O

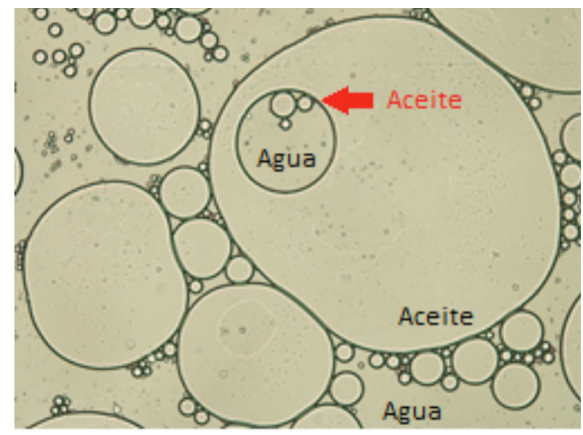

b) Múltiple $\mathrm{O} 1 / \mathrm{W} 1 / \mathrm{O} 2 / \mathrm{W} 2$

Figura 2. Tipos de a emulsión de acuerdo a la complejidad. Nota: Fuente propia del estudio.

Recientemente, las formulaciones basadas en lípidos han surgido como una de las mejores y más efectivas soluciones para administrar medicamentos poco solubles, entre los cuales, los sistemas de administración de fármacos auto nanoemulsificantes, han demostrado ser una tecnología prometedora para mejorar la biodisponibilidad sistémica oral de drogas o fitoconstituyentes que son poco solubles en agua, proporcionando a 
Tabla 1. Matriz de análisis par a muestras a diferente composición y HLB para mezclas Span85/Tween85.

\begin{tabular}{|c|c|c|c|c|}
\hline Lote & Parafina & Agua & Surfactante & HLB \\
\hline \multirow{5}{*}{1} & \multirow{5}{*}{$85 \%$} & \multirow{5}{*}{$10 \%$} & \multirow{5}{*}{$5 \%$} & 6 \\
\hline & & & & 7 \\
\hline & & & & 8 \\
\hline & & & & 9 \\
\hline & & & & 10 \\
\hline \multirow{5}{*}{2} & \multirow{5}{*}{$87 \%$} & \multirow{5}{*}{$8 \%$} & \multirow{5}{*}{$5 \%$} & 6 \\
\hline & & & & 7 \\
\hline & & & & 8 \\
\hline & & & & 9 \\
\hline & & & & 10 \\
\hline \multirow{5}{*}{3} & \multirow{5}{*}{$87 \%$} & \multirow{5}{*}{$10 \%$} & \multirow{5}{*}{$3 \%$} & 6 \\
\hline & & & & 7 \\
\hline & & & & 8 \\
\hline & & & & 9 \\
\hline & & & & 10 \\
\hline
\end{tabular}

Nota: Fuente propia del estudio.

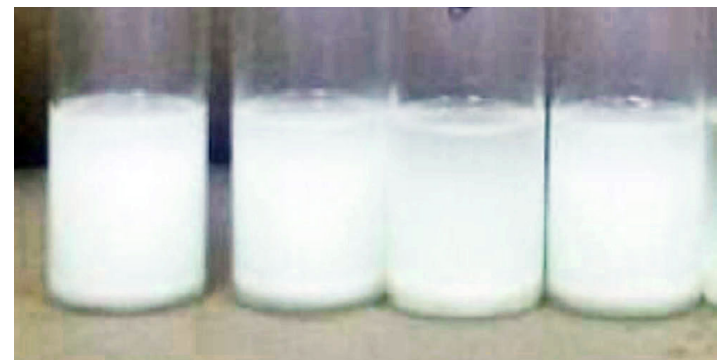

Figura 3. Sistema O/W/S relación 85/10/5 a HLB 13. De derecha a izquierda 1) Span 20 y Tween 80, 2) Span 80 y Tween 80 , 3) Span 85 y Tween 80 4) Span 85 y Tween 85) Nota: Fuente propia del estudio. 
su vez, una formulación con una biodisponibilidad mejorada, así como una estabilidad adecuada. Por lo tanto, las pequeñas gotas de aceite de las nanoemulsiones resultantes que se encuentran dispersas en los fluidos gastrointestinales, proporcionan una gran área interfacial para la absorción, aumentando así la actividad y minimizando la irritación debida al contacto del fármaco en la pared intestinal (Avachat y Patel, 2014; Hasan, 2014; Nazzal y Khan, 2002).

No obstante, la aplicación en la industria agrícola, no ha sido tan estudiada, siendo de gran importancia tener emulsiones autoemulsificables, ya que es conveniente realizar los procesos de mezclado en el campo de trabajo. Además, en el caso de pequeños agricultores, no se cuenta con equipo sofisticado, por ende, debe ser fácil de trabajarlo.

Otra de las ventajas de aplicar las emulsiones en este campo de estudio es que dan homogeneidad a la muestra y no dañan el equipo que se emplea para la aplicación, pues cuando se tiene materiales sólidos en suspensión tienen a tener bloqueos en los orificios de los aspersores.

Por lo tanto, en este estudio se pretende formular emulsiones estables de aceite en agua con capacidad de autoemulsicarse, utilizando un método de baja energía para que ocurra la inversión de la emulsión. Sin embargo, es importante considerar que la reproducibilidad y escalamiento de este tipo de tecnología deben ser estrictamente monitoreados.

\section{MATERIALES Y MÉTODOS}

\subsection{Materiales}

Todos los reactivos empleados fueron marca Merck con 99\% de pureza. Para la realización de emulsiones se empleó parafina líquida, agua destilada y de surfactantes, span 20, span 80, span 85, tween 20, tween 40, tween 60, tween 80 y tween 85 .

\subsection{Metodología}

\subsubsection{Prueba de combinación de surfactantes:}

Se procedió a realizar una mezcla de los surfactantes a HLB fijo. Lo anterior se realizó con la finalidad de ubicar la mejor combinación posible entre las diferentes líneas de tween y span, debido a que al combinar estos dos surfactantes se da un mejor equilibrio para posterior inversión de la emulsión, pues el tween posee características hidrofílicas (estabiliza emulsiones de W/O), mientras que el span posee características hidrofóbicas (estabiliza emulsiones de O/W). Por lo tanto, se realizó un total de 15 combinaciones posibles. En este caso se empleó un HLB de 13 para estudiar el sistema. La escogencia del sistema de surfactantes se realizó al observar la estabilidad a los 2 días posteriores de su elaboración. Para ello se denotó aparición de flotación y sedimentación. 


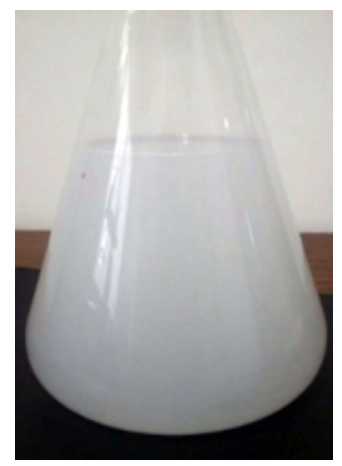

Figura 4. Dispersión en agua del Tween 85 y Span 85 (5mL de emulsión en $1 \mathrm{~L}$ agua) Nota: Fuente propia del estudio.

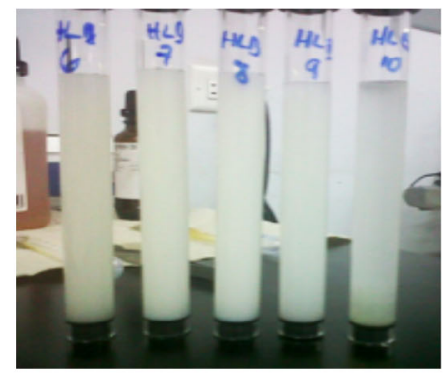

a) Lote 1. Muestras en tiempo 0

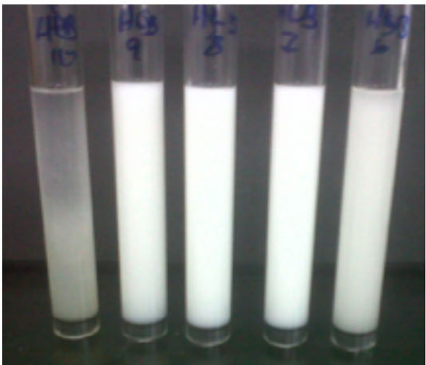

c) Lote 2. Muestras en tiempo 0

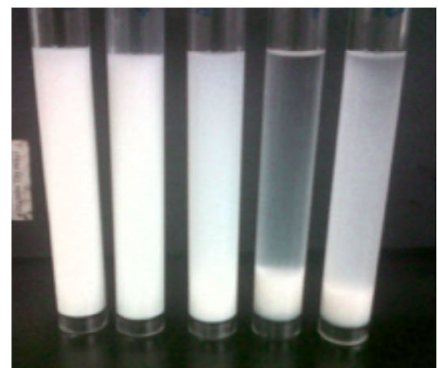

e) Lote 3. Muestras en tiempo 0

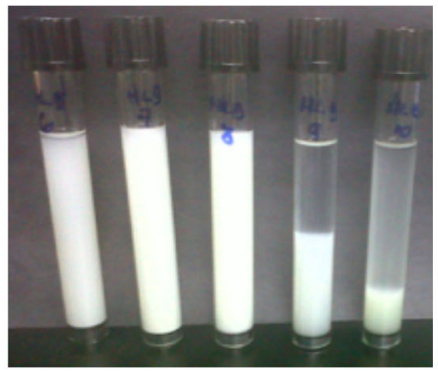

b) Lote 1. Muestras a 2 días preparación

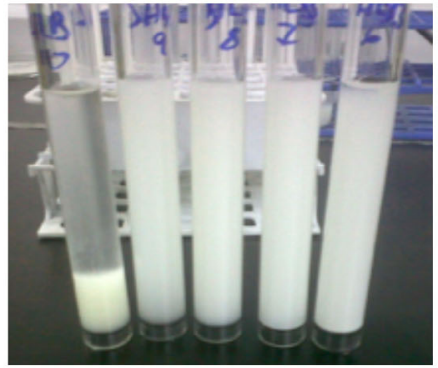

d) Lote 2. Muestras a 2 días preparación

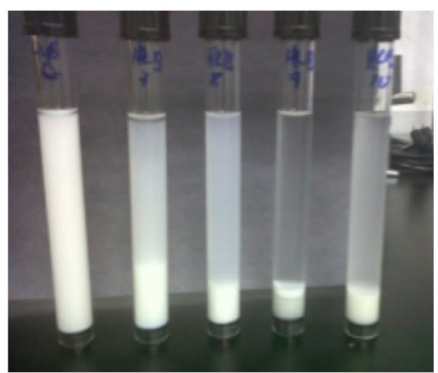

f) Lote 3 . Muestras a 2 días preparación

Figura 5. Muestras de los diferentes lotes con HLB 6, 7 ,8, 9 y 10. Nota: Fuente propia del estudio. 


\subsubsection{Formulación de emulsiones:}

Se realizó un barrido de composición con diferentes HLB (Tabla 1), según la combinación de surfactantes escogidos en la prueba anterior, siendo elegida la combinación de Span85/Tween85. El método de mezclado usado fue de baja energía, se utilizó un agitador magnético para la combinación de todos los componentes.

\subsubsection{Caracterización}

\subsubsection{Tamaño de partícula}

Se procedió a hacer una dilución de la emulsión en hexano (esto debido a que todas poseían fase externa parafina lo cual imposibilita la medición por láser), el cual se midió en el microtracnanotrac serie u2250 con el determinador de partículas con difracción de láser. Mientras que para la inversión de la emulsión O/W luego de la autoemulsificación se empleó el Mastersizer 2000, serie MAL 1051095. La diferencia en el empleo de los equipos se debe al rango de medición que posee cada uno.

\subsubsection{Estabilidad}

Se estudió la estabilidad del sistema con respecto al contenido de agua y al contenido de surfactante, siempre estudiando cada sistema por su HLB. Para determinar la estabilidad se procedió a medir a los dos días, el volumen de emulsión separado del volumen total de muestra. Por ende, se mide el índice deflotación y el de sedimentación. Al realizar esta relación se obtiene con ello un porcentaje de estabilidad, el cual representa la fracción de la emulsión que está estable.

\section{RESULTADOS Y DISCUSIÓN}

Al realizar la prueba de combinación de surfactantes se procedió a mezclar las líneas de tween y span para conocer cuál de las combinaciones posibles muestra mayor estabilidad y capacidad de autoemulsificación.

En la Figura 3 se obtiene que el tubo 4 (de izquierda a derecha) posee la mezcla de surfactantes más estables, siendo esta mezcla la de Span 85 y Tween 85. Al realizar la prueba de autoemulsificación en agua, esta da positiva sin necesidad de mucha agitación. Tal y como se puede observar en la figura 4, no presenta turbidez, separaciones y se ve homogénea.

Al obtener estos resultados, se procedió a buscar una formulación en donde se mejore su estabilidad, es por ello que se realizó otro barrido de formulación. No obstante, en estos casos se busca cambiar HLB (Balance Lipofílico-Hidrofílico) variando las proporciones de los surfactantes. Este barrido de formulación se realizó con HLB desde 10 hasta 6 con las mezclas de surfactante tween 85 y span 85 , según como lo muestra la Tabla 1. Los resultados se pueden ver en la Figura 5, donde se observan las emulsiones a la hora de realizarse en el tiempo 0 y luego de dos días de haberse formulado. En estas 


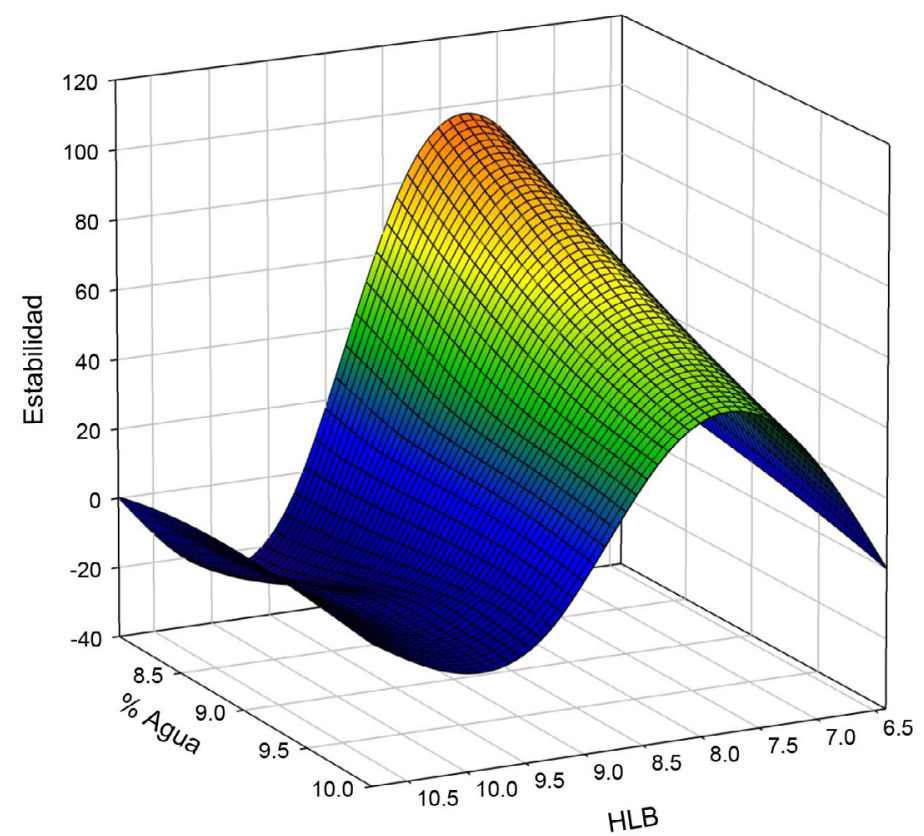

Figura 6. Variación de la estabilidad con respecto al contenido de agua y HLB. Nota: Fuente propia del estudio.

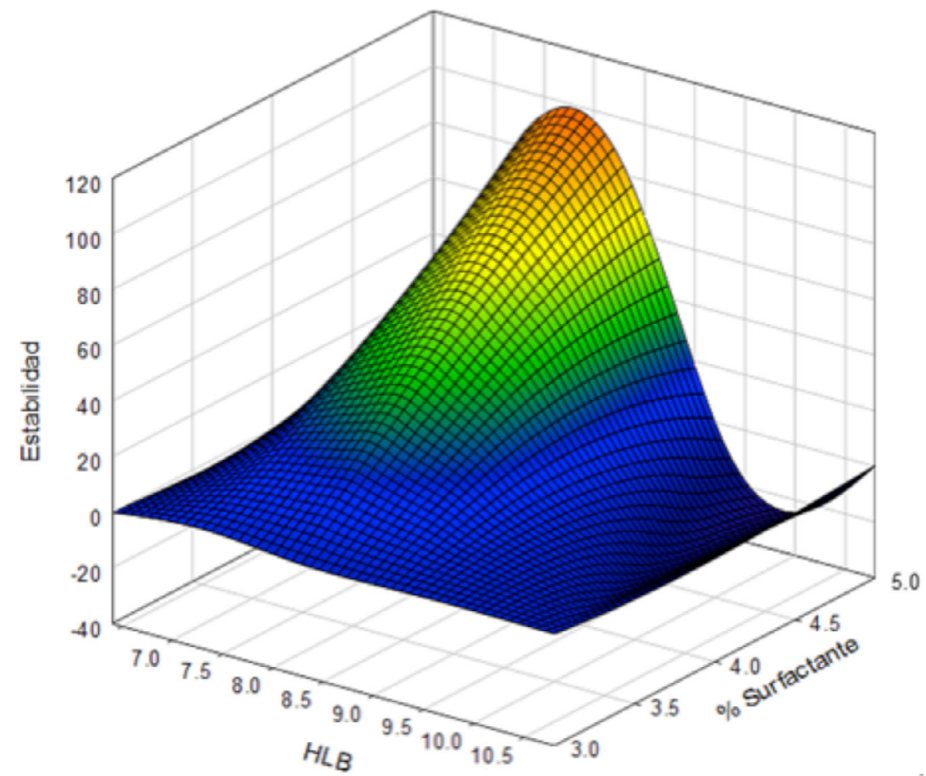

Figura 7. Variación de la estabilidad con respecto al contenido de surfactante y HLB. Nota: Fuente propia del estudio. 
imágenes se muestra cómo emulsiones que no son estables tienden a separarse luego de esos dos días, de tal forma que presentan sedimentación o flotación.

Por una parte, cuando se observa el lote tres, este presenta las emulsiones más inestables, pues solamente una emulsión (HLB de 6) muestra estabilidad con el tiempo. Por otra parte, el lote uno presenta tres emulsiones estables (HLB 6, 7 y 8); mientras que el lote dos presenta cuatro emulsiones estables (HLB 6, 7, 8, 9). No obstante, al comparar las emulsiones estables del lote uno y del lote dos se obtiene que es más viscosa y posee un menor tamaño de gota, indicando con ello mejor estabilidad con el tiempo.

Con los datos anteriores se procedió a realizar las medidas de estabilidad (porcentaje de estabilidad, medido como fracción de emulsión estable entre el total de la emulsión). Con ello se realizan las gráficas de porcentaje de agua o porcentaje de surfactante para cada HLB medido, obteniendo con ellos las Figura 6 y 7. Estas gráficas permiten estudiar la estabilidad de todo el sistema y predecir el comportamiento en otro de los puntos del mapa mostrado.

Con el barrido de formulación a diferentes HLB, se logró determinar la estabilidad de las muestras, observando que a un HLB de 8 y un porcentaje de agua mínimo, las formulaciones elaboradas tuvieron una mayor estabilidad (Figura 6) al igual que cuando se aumentó la concentración de surfactante con el mismo valor de HLB (Figura 7).

Por otra parte, se llevó a cabo la caracterización del lote uno, pues es el que presenta mayor estabilidad. Se decidió medir el tamaño de gota de las emulsiones a diferentes HLB, estas son emulsiones de agua/aceite (Figura 8). Posteriormente, se realizó la autoemulsificación o dispersión, de cada una de estas emulsiones, en agua (emulsiones de aceite/agua por la inversión de la emulsión) y se procedió a medir el tamaño de gota. Al obtener estos dos comportamientos para cada HLB se logró denotar cuál de ellas presenta el menor tamaño de gota, y por consiguiente estabilidad.

En cuanto a las emulsiones iniciales de agua/acetite se encontró que conforme aumentaba el HLB del surfactante, el tamaño de gota aumentaba, este comportamiento se ve en los HLB de 6, 7 y 8; así, luego de este valor se mantiene el tamaño de gota (Tabla 2 y Figura 8). Mientras, que al realizar la autoemulsificación (autodispersión en agua de las emulsiones iniciales), se obtiene un comportamiento bimodal para las combinaciones de HLB de 6, 8 y 9, siendo de menor tamaño de gota la de HLB de 8. Con ello, se demostró que esta es la que presenta mayor estabilidad (Tabla 2 y Figura 9).

En la Figura 10, se muestra la microscopia de las emulsiones del lote 1 para HLB extremos. En donde se comprueba que posee menor tamaño de gota las emulsiones con HLB de7. 


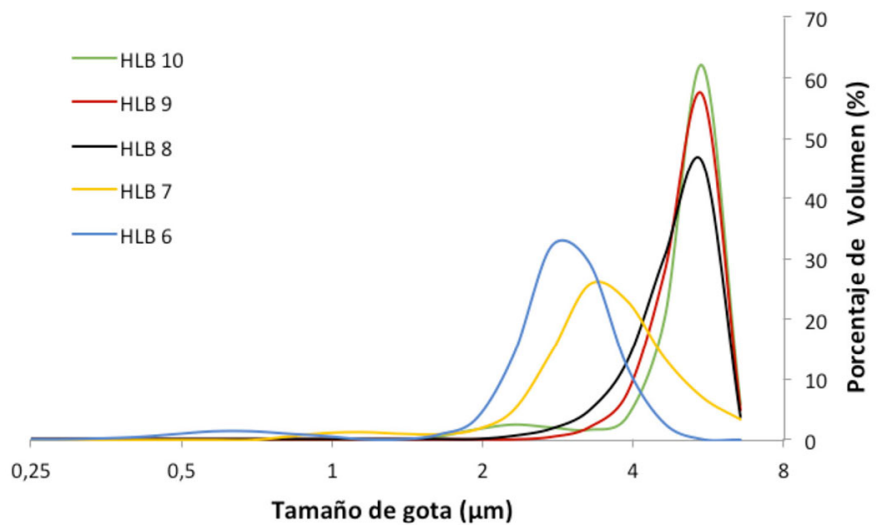

Figura 8. Variación del tamaño de gotas de la emulsión de agua en aceite a diferentes HLB Nota: Fuente propia del estudio.

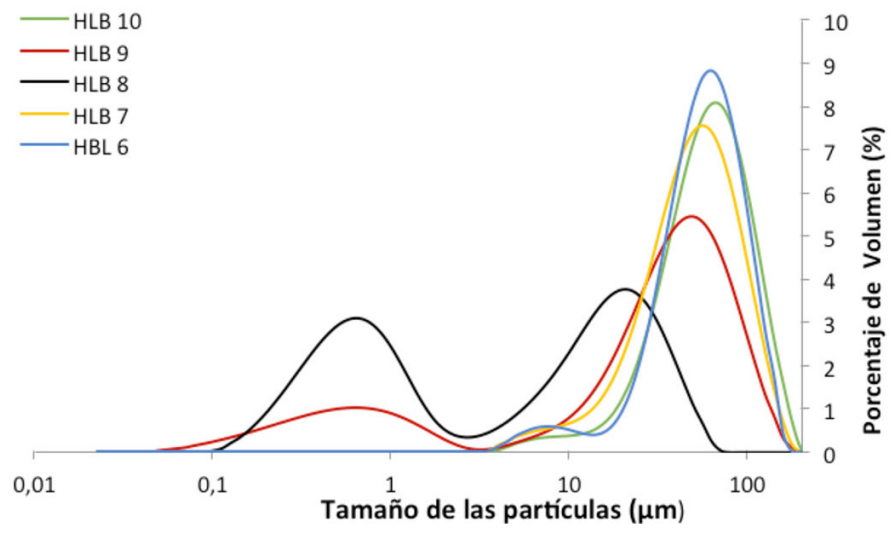

Figura 9. Variación del tamaño de gota para la emulsión de aceite en agua, (Inversión de la emulsión cuando se autoemulsifica en agua) a diferentes HLB. Nota: Fuente propia del estudio.

Tabla 2. Tamaño promedio de las gotas en las emulsiones.

\begin{tabular}{ccc}
\hline \multirow{2}{*}{ HLB } & \multicolumn{2}{c}{ Tamaño de gota $(\mu \mathrm{m})$} \\
\cline { 2 - 3 } & Agua / Aceite & Inversión Agua / Aceite \\
\hline 6 & 2,75 & $7,01-63,24$ \\
7 & 3,37 & 53,37 \\
9 & 4,62 & $0,63-22,44$ \\
9 & 5,50 & $0,71-50,23$ \\
10 & 5,50 & 70,96 \\
\hline
\end{tabular}




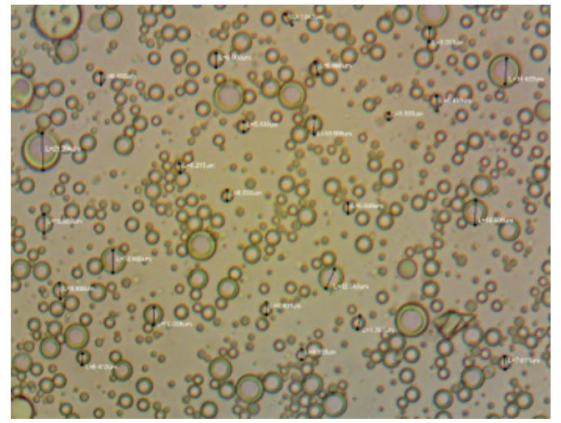

a) Lote 1 , HLB 9

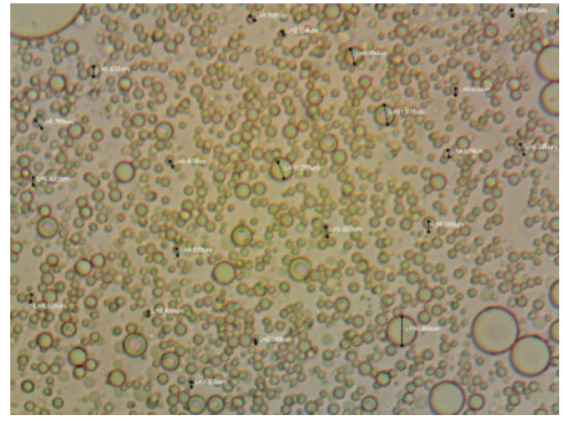

b) Lote 1 , HLB 7

Figura 10. Microscopia de las emulsiones de agua/aceite para diferentes emulsiones. Magnificación de 40X.

\section{CONCLUSIONES}

- $\quad$ En las emulsiones de agua/aceite se obtiene que son más estables las emulsiones de mezclas de twen 85 y span 85 , con un HLB de 6.

- Con los sistemas estudiados para las emulsiones autoemulsificables (aceite/agua) se obtiene que son más estables son las que poseen altas cantidades de surfactante, un porcentaje de agua menor a 9,5\% y un HLB de 8 .

- Se logró realizar una emulsión de agua/aceite estable que tenga la capacidad de autoemulsificarse al entrar en contacto con el agua sin necesidad de agitación, generando una inversión de la emulsión a aceite/agua.

- Es importante seguir trabajando con estos sistemas debido a la variedad de aplicaciones que se han venido desarrollando en los últimos años en las áreas farmacéutica, alimenticia y cosmética.

\section{REFERENCIAS}

Avachat, A. M., y Patel, V. G. (2014). Self nanoemulsifying drug delivery system of stabilized ellagic acid - phospholipid complex with improved dissolution and permeability. Saudi Pharmaceutical Journal, 23(3), 276-289. https://doi.org/10.1016/j.jsps.2014.11.001

Hasan, N. M. Y. (2014). Role of medium-chain fatty acids in the emulsification mechanistics of self-micro-emulsifying lipid formulations. Saudi Pharmaceutical Journal, 22(6), 580-590. https://doi.org/10.1016/j.jsps.2014.02.005

Melik, D.H; y Fogler, H.S (1988). Fundamentals of colloidal stability in quiesant media. Enciclopedia of emulsion technology. Bacher P. Marcel Dekke,inc,New York.

Morrison, D., y Ross, S. (2002). Emulsions. Colloidal dispersions suspensions, emulsions and foams. New York: John Wiley and Sons, Inc.

Muguet, V., Seiller, M., Barratt, G., Ozer, O., Marty, J. P., y Grossiord, J. L. (2001). Formulation of shear rate sensitive multiple emulsions. Journal of Controlled Release, 70(1-2), 37-49. https:// doi.org/10.1016/S0168-3659(00)00314-X 
Nazzal, S., y Khan, M. A. (2002). Response surface methodology for the optimization of ubiquinone self-nanoemulsified drug delivery system. AAPS PharmSciTech, 3(1), 23-31. https://doi. org/10.1208/pt030103

Piorkowski, D. T., y McClements, D. J. (2013). Beverage emulsions: Recent developments in formulation, production, and applications. Food Hydrocolloids, 42, 5-41. https://doi.org/10.1016/j. foodhyd.2013.07.009

Salager, J. (1999). Formulación, Composición y Fabricación de Emulsiones para Obtener las Propiedades deseadas. Estado del Arte Parte A: Introducción y Conceptos de Formulación Fisicoquímica. Mérida, Venezuela: Laboratorio de Formulación, Interfases Reologia y Procesos (FIRP). Universidad de los Andes.

Tamilvanan, S. (2004). Progress in Lipid Research Oil-in-water lipid emulsions : implications for parenteral and ocular delivering systems. Progress in Lipid Research, 43, 489-533. https:// doi.org/10.1016/j.plipres.2004.09.001

Verbeken, D. (2005). Rheology of Emulsions. In Advances in Food Rheology and its Applications (pp. 437-457). Turkey: Elsevier Ltd. https://doi.org/10.1016/B978-0-08-100431-9/00017-6

Weiner, Norman. (1974). Emulsions and emulsion technology, part I. K.J Lissant, ed. New York. Surfactant Science Series. 440 pp 